\title{
LI-RADS Classification and Outcomes of Hepatocellular Carcinoma Treated with Transcatheter Arterial Chemoembolization Combined with Radiofrequency Ablation
}

\section{Yuji Tachibana}

Graduate School of Welfare and Health Sciences, Oita University

\section{Ryo Takaji}

Department of Radiology, Faculty of Medicine, Oita University

\section{Miyuki Maruno}

Department of Radiology, Faculty of Medicine, Oita University

Koichi Honda

Department of Gastroenterology, Faculty of Medicine, Oita University

Mizuki Endo

Medical Safety Management Center, Oita University Hospital

\section{Kazunari Murakami}

Department of Gastroenterology, Faculty of Medicine, Oita University

Yoshiki Asayama ( $\square$ asayama@oita-u.ac.jp)

Department of Radiology, Faculty of Medicine, Oita University

\section{Research Article}

Keywords: Hepatocellular Carcinoma, Transcatheter Arterial, Radiofrequency Ablation

Posted Date: December 16th, 2021

DOl: https://doi.org/10.21203/rs.3.rs-1144653/v1

License: (a) (i) This work is licensed under a Creative Commons Attribution 4.0 International License. Read Full License 


\section{Abstract}

\section{Purpose}

The aim of this study was to clarify the usefulness of the Liver Imaging Reporting and Data System (LIRADS) to predict the patients' prognosis after transcatheter arterial chemoembolization (TACE) combined with radiofrequency ablation (TACE-RFA) for hepatocellular carcinoma (HCC) of Barcelona-Clinic Liver Cancer Stage (BCLC) 0 or A.

Materials and Methods

We retrospectively analyzed cases of HCC patients who underwent TACE-RFA (Jan 2005 to Dec 2015). Patients' nodules were categorized based on their LI-RADS v2018 core. The LI-RADS category was assigned to each nodule using dynamic contrast-enhanced CT. LR-3, LR-4 and LR-5 nodules were extracted. We assessed the overall survival (OS) and recurrence-free survival (RFS) among BCLC 0 and BCLC A patients.

Results

Of the 64 nodules extracted, 22 were LR-3 or $-4(14.8 \pm 6.7 \mathrm{~mm})$ and 42 were LR-5 $(17.1 \pm 6.9 \mathrm{~mm})$. Regarding OS, there was no significant difference between LR-3 or -4 and LR-5 ( $p=0.278)$. In terms of RFS, there was a significant difference between LR-3 or -4 and LR-5 $(p=0.03)$. In particular, patients with BCLC A with LR-5 nodules showed significantly poorer RFS than those with LR-3 or $-4(p=0.016)$ nodules.

Conclusions

For patients with BCLC A, LR-3 or -4 are associated with a better prognosis than LR-5 nodules.

\section{Introduction}

Hepatocellular carcinoma (HCC) is the third leading cause of cancer-related death worldwide, accounting for $85-90 \%$ of primary liver tumors [1-3]. HCC treatment depends on the progression, and prognosis can also vary significantly $[4,5]$. Therefore, early detection of HCC is very important. Currently, HCC can be diagnosed non-invasively by image analysis.

The American Association of Radiologists has developed a standardized diagnostic algorithm termed the Liver Reporting and Data System (LI-RADS) to classify HCC using images such as dynamic multidetector computed tomography (CT), dynamic magnetic resonance imaging (MRI), and ultrasonography (US), together with other findings [6]. LI-RADS can be used to make a detailed diagnosis by understanding the patient's tumor condition, which may be useful for predicting prognosis and selecting treatment methods [7]. 
Prognosis remains poor for patients with advanced HCC. Patients diagnosed early, however, are eligible for curative treatment such as surgical resection, liver transplantation, and radiofrequency ablation (RFA) [8]. In addition, treatment with transcatheter arterial chemoembolization (TACE) may be performed for HCC, and there are many reports that TACE combined with RFA (TACE-RFA) has even better treatment results than TACE treatment alone [9-11]. While there are many reports on the treatment results of TACERFA, few studies have considered the relation between the prognosis of patients who underwent TACERFA and the LI-RADS of the target tumor, and the details remain to be clarified.

The purpose of this study was to assess the relation between LI-RADS grading and the treatment effect of TACE-RFA, and to clarify the prognosis of TACE-RFA treatment in patients with early-stage HCC of Barcelona-Clinic Liver Cancer Stage (BCLC) 0 and A.

\section{Materials And Methods}

\section{Study design and patients}

This was a retrospective study approved by the review board at our institution. The requirement for informed consent was waived. We retrospectively analyzed cases of HCC patients who underwent TACERFA between January 2005 and December 2015. A total of 562 patients were diagnosed with liver tumor by diagnostic imaging modalities such as CT, MRI and US during this period, and subsequently underwent TACE. Of these, 276 patients had no history of treatment-including TACE, RFA, or resectionand thus were receiving their first treatment for HCC. In this subgroup, 73 patients had TACE on the all tumors and RFA on the same tumors within a week. In addition, 69 of these patients had had three-phase dynamic CT images taken before TACE, and 66 of these patients had BCLC 0 and A; the latter group comprised the subjects of this study. The extracted patients were divided by BCLC stage according to the Bolondi classification $[12,13]$. For all nodules, images obtained by postoperative CT confirmed the deposition of Lipiodol.

Nodules were classified according to the LI-RADS category using CT imaging. Among patients with BCLC 0 and A stage cancer, those with nodules classified as LR-3 (intermediate probability of being HCC), LR-4 (probably HCC) or LR-5 (definitely HCC) were extracted. In this assessment, patients with nodules classified as LR-M, i.e., probably or definitely malignant but not HCC specific, were excluded. Our final subject pool included 64 patients with LR-3, -4 and -5 tumors (Fig. 1).

If one patient was being treated for multiple tumors, we considered the largest one. Patient age ranged from 35-91 years (mean 71.9). The male-female ratio was 37 males (58\%) to 27 females (42\%). Other characteristics of patients are listed in Table 1. 


\section{Characteristic}

\begin{tabular}{|c|c|c|}
\hline Age $(y)$, mean $\pm S D$ & & $71.9 \pm 9.8$ \\
\hline \multirow[t]{2}{*}{ Sex } & Male & 37 \\
\hline & Female & 27 \\
\hline AFP (ng/mL), mean (range) & & $44.7(1.6-982.7)$ \\
\hline PIVKA-II (mAU/mL), mean (range) & & $579.4(10-20836)$ \\
\hline \multirow[t]{2}{*}{ Child-Pugh } & $A$ & 50 \\
\hline & B & 14 \\
\hline \multirow[t]{2}{*}{ BCLC } & 0 & 31 \\
\hline & A & 33 \\
\hline \multirow[t]{3}{*}{ LI-RADS } & 3 & 3 \\
\hline & 4 & 19 \\
\hline & 5 & 42 \\
\hline \multirow[t]{2}{*}{ Anti-cancer drug } & Epirubicin & 56 \\
\hline & Miriplatin & 8 \\
\hline Tumor size $(\mathrm{mm})$, mean $\pm S D$ & & $16.1 \pm 7.0$ \\
\hline
\end{tabular}

We then assessed the overall survival (OS) and recurrence-free survival (RFS) between the LR-3 or -4 and LR- 5 cases, and between BCLC 0 and BCLC A patients.

\section{CT protocol}

CT scans were performed at our hospital and related hospitals using a 64-section multidetector CT (MDCT) scanner (Aquilion CX TSX-101A/NA; Canon Medical Systems, Tochigi, Japan) or a 320-section MDCT scanner (Aquilion ONE TSX-301A/2A; Canon Medical Systems, Tochigi, Japan). The scanning parameters used for MDCT were as follows: $120 \mathrm{kVp}, 200-400 \mathrm{mAs}$, rotation time $0.5 \mathrm{~s}$, pitch of 0.98 (64 detectors) and 0.6 (320 detectors), and section thickness of $1 \mathrm{~mm}$ with a $1 \mathrm{~mm}$ reconstruction interval. CT images were acquired via image archives and communication systems.

For three-phase CT imaging, a total of $100 \mathrm{~mL}$ of contrast medium (Iopamiron 370; Bayer Schering Pharma, Leverkusen, Germany) was injected using a power injector at a rate of $3 \mathrm{~mL} / \mathrm{s}$. Each scan delay 
was determined using automatic bolus tracking. Images of three phases (arterial phase, portal vein phase, and equilibrium phase) were acquired.

\section{Transcatheter arterial chemoembolization}

TACE was performed by at least one of two board certificated interventional radiologists (R.T and M.M with 12 and 10 years). In interventional radiology, we used digital subtraction angiography, CT during hepatic arteriogram (CTHA), and CT during arterial portography (CTAP) to assess the number and size of tumors and identify tumor-feeding vessels. Once the trophic arteries of the target tumor nodule were identified by this method, TACE was performed using anticancer drugs, poppy seed oil (Lipiodol; Guerbet Japan, Tokyo), and gelatin particles. As an anticancer drug, 10-60 mg epirubicin (Farmorubicin, Kyowa Hakko Kogyo, Tokyo) or 60-120 mg miriplatin (Miripla, Sumitomo Dainippon Pharma Co., Osaka, Japan) was used with iodine. The amounts of the drugs were determined by a consensus between radiologists and gastroenterologists. Anticancer drugs were mixed with 2-10 ml poppy oil and injected via a microcatheter. Gelatin particles (Gelpart; Nippon Kayaku, Tokyo) were then injected until the feeding arteries were completely embolized.

\section{Radiofrequency ablation}

All patients were treated with monopolar RFA by at least one of two board certificated gastroenterologists (K.H and M.E with 13 and 6 years of experience). RFA was performed under US guidance within three days to one week of TACE treatment. In RFA, we used a 17-gauge internally cooled electrode with a 2 or 3 $\mathrm{cm}$ exposed tip (Cool-tip; Radionics, Burlington, MA). When using a $2 \mathrm{~cm}$ needle, the output was started at $40 \mathrm{~W}$ and increased by $10 \mathrm{~W}$ every min for incineration. For a $3 \mathrm{~cm}$ needle, the output was started at $60 \mathrm{~W}$ and increased by $10 \mathrm{~W}$ every min for incineration. In both cases, incineration continued until the output rolled off.

\section{LI-RADS category}

We categorized the nodules for analysis according to the LI-RADS v2018 core [7]. The LI-RADS category was assigned to each nodule using dynamic contrast-enhanced CT. If two or more nodules were evaluated, we selected the largest. Major imaging features used in the classification included arterial phase hyperenhancement, observation size, washout, and capsule appearance. Patients were also categorized with hepatic observation threshold growth added to the extent possible. In addition, subfindings were used to fine-tune the categorization (upgrade the category if there were findings supporting malignancy; downgrade it if there were findings supporting benignity). If we were uncertain about the categorization, we used tie-breaking rules that would bring us one step closer to LR-3. Images were evaluated by board certificated radiographer (Y.T with 14 years of experience) and board certificated radiologist (Y.A with 19 years of experience) according to these rules.

\section{Statistical analysis}

We evaluated OS and RFS for all patients. BCLC 0 and A were extracted according to the Bolondi classification, and OS and RFS were compared by LI-RADS category. OS was defined as the time from the 
day of TACE treatment to the day of death. RFS was defined as the time from the date of TACE treatment to the date of tumor recurrence or death. Patients who remained alive at the date of the last follow up were censored in the statistical analysis. For assessment of recurrence, three-phase CT and dynamic MRI were used. Recurrence was defined when tumors that showed arterial phase enhancement and washout appeared locally or elsewhere in the liver. The observation period was 1-116 months.

Regarding OS and RFS, the whole was divided into two groups, and each item was compared. Fisher's exact test was used for comparison between the two groups. The boundaries were 70 years old, alfafetoprotein (AFP) $10 \mathrm{ng} / \mathrm{mL}$, and protein induced by vitamin K absence or antagonist-II (PIVKA-II) 40 $\mathrm{mAU} / \mathrm{mL}$. Comparisons were made between the two groups $A$ and $B$ for Child-Pugh score, and between the two groups A and 0 for BCLC. Regarding LI-RADS, we again compared two groups, LR-3 or 4 and LR-5. These items were compared using Cox regression analysis. Also, survival was assessed by the KaplanMeier method and log-rank test. Variables with $p<0.2$ at univariate analysis were applied multivariate analysis to identify the most reliable prognostic marker. The evaluation was done using R (version 4.0.3; The R Foundation).

\section{Results}

Univariate analysis and multivariate analysis of OS was performed on the extracted 64 patients (Table 2). We also performed univariate analysis and multivariate analysis of RFS (Table 3). There was a significant difference only in the comparison of LI-RADS regarding RFS. For other items, no significant difference was found in both univariate and multivariate analysis. 
Table 2

Univariate Analyses and Multivariate Analyses of Factors Affecting Overall Survival Univariate Analyses Multivariate Analyses

\begin{tabular}{|c|c|c|c|c|c|c|c|}
\hline \multirow[b]{2}{*}{ Variable } & \multirow[b]{2}{*}{ Cases } & \multicolumn{3}{|c|}{ Univariate Analyses } & \multicolumn{3}{|c|}{ Multivariate Analyses } \\
\hline & & $\mathrm{HR}$ & $95 \% \mathrm{Cl}$ & $p$ & $\mathrm{HR}$ & $95 \% \mathrm{Cl}$ & $p$ \\
\hline Age (>70 vs $\leq 70)$ & $\begin{array}{l}40 \text { vs } \\
24\end{array}$ & 1.81 & $\begin{array}{l}0.76- \\
4.31\end{array}$ & 0.184 & 1.27 & $\begin{array}{l}0.42- \\
3.80\end{array}$ & 0.675 \\
\hline Sex (Male vs Female) & $\begin{array}{l}37 \text { vs } \\
27\end{array}$ & 1.75 & $\begin{array}{l}0.77- \\
3.98\end{array}$ & 0.181 & 0.85 & $\begin{array}{l}0.30- \\
2.47\end{array}$ & 0.771 \\
\hline $\operatorname{AFP}(>10$ vs $\leq 10)$ & $\begin{array}{l}25 \mathrm{vs} \\
34\end{array}$ & 2.11 & $\begin{array}{l}0.89- \\
5.003\end{array}$ & 0.091 & 2.49 & $\begin{array}{l}0.78- \\
7.99\end{array}$ & 0.124 \\
\hline PIVKA (>40 vs $\leq 40)$ & $\begin{array}{l}18 \text { vs } \\
39\end{array}$ & 1.89 & $\begin{array}{l}0.74- \\
4.82\end{array}$ & 0.183 & 2.55 & $\begin{array}{l}0.89- \\
7.37\end{array}$ & 0.083 \\
\hline Child-Pugh score (A vs B) & $\begin{array}{l}50 \text { vs } \\
14\end{array}$ & 0.50 & $\begin{array}{l}0.21- \\
1.20\end{array}$ & 0.121 & 0.79 & $\begin{array}{l}0.22- \\
2.84\end{array}$ & 0.720 \\
\hline BCLC (0 vs A) & $\begin{array}{l}31 \text { vs } \\
33\end{array}$ & 2.02 & $\begin{array}{l}0.82- \\
4.97\end{array}$ & 0.128 & 1.68 & $\begin{array}{l}0.56- \\
5.08\end{array}$ & 0.357 \\
\hline $\begin{array}{l}\text { Anti-cancer drug (Epirubicin vs } \\
\text { Milliplatin) }\end{array}$ & $\begin{array}{l}56 \mathrm{vs} \\
8\end{array}$ & 1.07 & $\begin{array}{l}0.36- \\
3.18\end{array}$ & 0.907 & & & \\
\hline LI-RADS (LR-3 or 4 vs LR-5) & $\begin{array}{l}22 \text { vs } \\
42\end{array}$ & 1.66 & $\begin{array}{l}0.66- \\
4.16\end{array}$ & 0.278 & 0.93 & $\begin{array}{l}0.33- \\
2.63\end{array}$ & 0.894 \\
\hline \multicolumn{8}{|c|}{$\begin{array}{l}\text { Abbreviations: HR: hazard ratio; AFP: alfa-fetoprotein; PIVKA-II: protein induced by vitamin K absence } \\
\text { or antagonist-II; BCLC: Barcelona Clinic Liver Cancer Stage; LI-RADS: Liver Imaging Reporting and } \\
\text { Data System. }\end{array}$} \\
\hline \multicolumn{8}{|l|}{ Data were available in 59 cases. } \\
\hline
\end{tabular}


Table 3

Univariate Analyses and Multivariate Analyses of Factors Affecting Recurrence-free Survival

\begin{tabular}{|c|c|c|c|c|c|c|c|}
\hline \multirow[b]{2}{*}{ Variable } & \multirow[b]{2}{*}{ Cases } & \multicolumn{3}{|c|}{ Univariate Analyses } & \multicolumn{3}{|c|}{ Multivariate Analyses } \\
\hline & & $\mathrm{HR}$ & $95 \% \mathrm{Cl}$ & $p$ & $\mathrm{HR}$ & $95 \% \mathrm{Cl}$ & $p$ \\
\hline Age $(>70$ vs $\leq 70)$ & $\begin{array}{l}40 \text { vs } \\
24\end{array}$ & 1.00 & $\begin{array}{l}0.55- \\
1.82\end{array}$ & 0.993 & & & \\
\hline Sex (Male vs Female) & $\begin{array}{l}37 \text { vs } \\
27\end{array}$ & 1.58 & $\begin{array}{l}0.87- \\
2.87\end{array}$ & 0.134 & 1.44 & $\begin{array}{l}0.72- \\
2.87\end{array}$ & 0.297 \\
\hline $\operatorname{AFP}(>10$ vs $\leq 10)$ & $\begin{array}{l}25 \text { vs } \\
34\end{array}$ & 1.44 & $\begin{array}{l}0.76- \\
2.72\end{array}$ & 0.259 & 1.53 & $\begin{array}{l}0.78- \\
3.00\end{array}$ & 0.217 \\
\hline PIVKA (>40 vs $\leq 40)$ & $\begin{array}{l}18 \text { vs } \\
39\end{array}$ & 1.23 & $\begin{array}{l}0.62- \\
2.44\end{array}$ & 0.559 & & & \\
\hline Child-Pugh score (A vs B) & $\begin{array}{l}50 \text { vs } \\
14\end{array}$ & 0.97 & $\begin{array}{l}0.47- \\
1.97\end{array}$ & 0.923 & & & \\
\hline BCLC (0 vs $A)$ & $\begin{array}{l}31 \text { vs } \\
33\end{array}$ & 1.43 & $\begin{array}{l}0.79- \\
2.59\end{array}$ & 0.241 & 1.37 & $\begin{array}{l}0.73- \\
2.58\end{array}$ & 0.324 \\
\hline $\begin{array}{l}\text { Anti-cancer drug (Epirubicin vs } \\
\text { Milliplatin) }\end{array}$ & $\begin{array}{l}56 \text { vs } \\
8\end{array}$ & 0.57 & $\begin{array}{l}0.22- \\
1.46\end{array}$ & 0.241 & 0.44 & $\begin{array}{l}0.15- \\
1.31\end{array}$ & 0.140 \\
\hline LI-RADS (LR-3 or 4 vs LR-5) & $\begin{array}{l}22 \text { vs } \\
42\end{array}$ & 2.05 & $\begin{array}{l}1.07- \\
3.93\end{array}$ & 0.030 & 1.69 & $\begin{array}{l}0.85- \\
3.36\end{array}$ & 0.132 \\
\hline \multicolumn{8}{|c|}{$\begin{array}{l}\text { Abbreviations: HR: hazard ratio; AFP: alfa-fetoprotein; PIVKA-II: protein induced by vitamin K absence } \\
\text { or antagonist-II; BCLC: Barcelona Clinic Liver Cancer Stage; LI-RADS: Liver Imaging Reporting and } \\
\text { Data System. }\end{array}$} \\
\hline \multicolumn{8}{|l|}{ [Data were available in 59 cases. } \\
\hline${ }^{\square}$ Data were available in 57 cas & & & & & & & \\
\hline
\end{tabular}

Of the 64 patients, BCLC classification resulted in 31 patients in the BCLC 0 group, and 33 in the BCLC A group. Patients with BCLC 0 and BCLC A were then extracted and classified by LI-RADS classification. In BCLC 0 patients, 1 tumor was LR-3, 12 were LR-4, and 18 were LR-5. For BCLC A patients, 2 tumors were LR-3, 7 were LR-4, and 24 were LR-5. Table 4 shows the baselines characteristics of BCLC 0 / A with LR-3 or 4, and LR-5 patients, which were the targets of this comparison. Example clinical images are presented in Fig. 2. Fig. 2a, 2b, and $2 c$ are images of a patient with a tumor corresponding to LR-4 at BCLC 0; Fig. 2d, $2 e, 2 f$, and $2 \mathrm{~g}$ are images of a patient with a tumor corresponding to LR-5 in BCLC A. 
Table 4

Baseline Characteristics of LR-3 or -4 and LR- 5 Patients

\begin{tabular}{|c|c|c|c|}
\hline Characteristic & LR-3 or -4 & LR-5 & $p$ \\
\hline Age $(y)$, mean $\pm S D$ & $70.0 \pm 9.1$ & $73.2 \pm 10.0$ & 0.159 \\
\hline Sex & & & 0.495 \\
\hline Male & 14 & 23 & \\
\hline Female & 8 & 19 & \\
\hline AFP (ng/mL) & & & 0.955 \\
\hline$>10$ & 9 & 16 & \\
\hline$\leq 10$ & 12 & 22 & \\
\hline no data & 1 & 4 & \\
\hline Mean (range) & $36.0(1.6-179.8)$ & $48.7(2.1-982.7)$ & 0.731 \\
\hline PIVKA-II (mAU/mL) & & & 0.048 \\
\hline$>40$ & 3 & 15 & \\
\hline$\leq 40$ & 17 & 22 & \\
\hline no data & 2 & 5 & \\
\hline Mean (range) & $28.2(10-117)$ & $877.2(14-20836)$ & 0.288 \\
\hline Child-Pugh class & & & 0.605 \\
\hline A & 18 & 32 & \\
\hline B & 4 & 10 & \\
\hline BCLC & & & 0.217 \\
\hline 0 & 13 & 18 & \\
\hline A & 9 & 24 & \\
\hline Anti-cancer drug & & & 0.073 \\
\hline Epirubicin & 17 & 39 & \\
\hline Miriplatin & 5 & 3 & \\
\hline Tumor size $(\mathrm{mm})$, mean \pm SD (range) & $14.8 \pm 6.7(8.0-28.1)$ & $18.0 \pm 6.2(10.0-33.4)$ & 0.199 \\
\hline
\end{tabular}

Abbreviations: HR: hazard ratio; AFP: alfa-fetoprotein; PIVKA-II: protein induced by vitamin $\mathrm{K}$ absence or antagonist-II; BCLC: Barcelona Clinic Liver Cancer Stage. 


\begin{tabular}{|llll|}
\hline Characteristic & LR-3 or -4 & LR-5 & $p$ \\
\hline 1 & 18 & 36 \\
2 & 4 & 4 \\
3 & 0 & 2
\end{tabular}

Abbreviations: HR: hazard ratio; AFP: alfa-fetoprotein; PIVKA-II: protein induced by vitamin $\mathrm{K}$ absence or antagonist-II; BCLC: Barcelona Clinic Liver Cancer Stage.

Baseline characteristics of LR-3 or -4 and LR-5 patients were shown in Table 4. Comparison of groups showed a significant difference regarding PIVKA-II. There were no significant differences in age, sex, AFP, Child-Pugh class, BCLC, anticancer drug, or tumor size.

\section{Overall survival}

In a comparison of the 64 cases, there was no significant difference in OS between LR-3 or -4 and LR-5 in univariate analysis ( $p=0.278$ ) (Table 2). Fig. 3 a shows the results of the Kaplan-Meier estimate. Further, for BCLC 0 and A, LR-3 or -4 and LR-5 were extracted and compared (Fig. 3b and 3c). There were no significant differences between LR-3 or 4 and LR-5 regarding the OS of the BCLC 0 or BCLC A cases ( $p=0.952,0.194$, respectively).

\section{Recurrence-free survival}

The next comparison of 64 cases revealed a significant difference between LR-3 or -4 and LR- 5 in terms of RFS in univariate analysis ( $p=0.03$ ) (Table 3 ). Multivariable analysis with the cox proportional hazards regression model revealed that LR-5 was independent prognostic factor $(p=0.03)$. The Kaplan-Meier estimate is shown in Fig. 4a. As with the OS comparison, for BCLC 0 and A, LR-3 or -4 and -5 were extracted and compared. The results of the Kaplan-Meier curve for each result are shown in Fig. $4 \mathrm{~b}$ and 4c. For the BCLC 0 cases, there were no significant differences between LR-3 or -4 and LR-5 ( $p=0.533)$. For the BCLC A cases, however, the log-rank test results showed significant differences between LR-3 or -4 and LR-5 ( $p=0.016)$. The 1-, 3-, and 5-year survival rates of LR-4 patients were $89 \%, 56 \%$, and $33 \%$, while those for LR-5 patients were $58 \%, 8 \%$, and $4 \%$, respectively.

\section{Discussion}

In this study, we examined the prognosis between patients with nodules classified as LR-3 or -4 and those with LR-5 tumors. There was a significant difference in RFS in the comparison of all 64 cases. In particular, for BCLC A, we found that LR-5 had a worse RFS than LR-3 or -4. In contrast, there was no significant difference in OS between LR-3 or -4 and LR-5 stage tumors.

The influence of tumor size might be considered. When determining the LI-RADS classification, if conditions such as arterial enhancement and washout are the same, we will divide by tumor size [7]. 
Larger tumors may have more vascular invasion of the tumor, and a higher probability of recurrence [1416]. In addition, with a large tumor, some portions of the tumor that are not visible in the image may not be treatable, which may be related to the treatment results $[17,18]$. It is possible that the drug did not reach the interior of the tumor with TACE, or that even if a sufficient safety margin were assumed, RFA did not sufficiently incinerate the tumor. Also, when the tumor has a capsule, the larger the size, the easier it is to infiltrate outside beyond the capsule, and thus the greater likelihood of metastasis $[19,20]$.

As indicated in Table 4, there were no significant differences in age, gender, or AFP values between patients with LR-3 or -4 and those with LR- 5 stage tumors. However, there was a significant difference in PIVKA-II values. There is an association between PIVKA-II values and tumor vascular invasion [21]. LR-5 tumors have high PIVKA-II values, and there might be microvascular invasion that is not visible on the pretreatment image. As a result, we considered that the risk of recurrence was high in our patients with high PIVKA-Il values with LR-5 stage tumors.

Regarding the washout, previous reports revealed that washout of hypervascular HCC occurred earlier as the histological grade advanced, the histological architecture got closer to pure trabecular HCC, and hypervascular HCCs with thicker tumor plates showed worse histological grade and earlier washout pattern $[22,23]$. Thus, clear washout may indicate an aggressive tumor, such as a moderately to poorly differentiated HCC with thicker tumor plates.

In contrast, there was no significant difference in OS. One of the possible reasons for this is that HCC can show multicentric development [24, 25]. Even if a tumor was treated completely, new HCC, not intrahepatic metastasis, can develop elsewhere in the liver, which significantly influences survival. Unfortunately, in many cases it is difficult to tell the difference between intrahepatic metastasis from the original tumor and a newly developed tumor. Other possible factors that affected patients' prognosis included worsening liver function and cirrhosis, and these effects may have been greater than the tumor aggressiveness itself [26].

In this study, there was no significant difference between LR-3 or -4 and LR- 5 in the BCLC 0 group. In the very early stage of $\mathrm{HCC}$, if a tumor is well treated, the subsequent prognosis may depend on the likelihood of developing another HCC in the liver, or on the deterioration of underlying liver function [27]. There may have been cases in which recurrence unrelated to the primarily treated tumor occurred.

This study had several limitations: (1) It was a retrospective study and selection bias which could have affected the results might therefore exist. (2) Data were collected from a single center, and samples from multiple regions were required for further validation. If we could have collected more cases, we would have been able to investigate in more detail. (3) Two anticancer drugs were used during TACE. There was no significant difference observed due to the difference in anticancer drugs, but the accuracy of the study might have been improved if comparisons could have been made under the same drug conditions [28]. (4) Patients with BCLC 0 or A might be good surgical candidates; however, our hospital traditionally favored less invasive therapy such as TACE-RFA. Thus, patients who were eligible for surgery may have been included [29]. 
The LI-RADS classification is a classification of certainly, which is a tool for classifying images according to their major characteristics. Detailed tumor characteristics such as degree of differentiation are not included, and may be classified using other algorithms. Examining the LI-RADS classification by adding more detailed features of the tumor can provide a more detailed prognosis. It has also been reported that the combination of MRI diffusion-weighted image analysis and LI-RADS can improve confidence [30]. In the future, by combining LIRADS with various factors, we will find it easier to predict the prognosis.

In conclusion, tumors categorized as LR- 5 have a worse RFS than those classified as LR-3 or -4 , especially in BCLC A. LI-RADS, originally created for use at the radiologist's discretion in diagnosis, can predict patient prognosis. Tumors classified as LR-5 by LI-RADS require more attention to the patient's course than tumors of other classifications.

\section{Declarations}

Compliance with ethical standards

Funding: No funding was received for this study.

Conflict of interest: The authors declare that they have no conflict of interest.

Ethical approval: All procedures performed in this study involving human participants were in accordance with the ethical standards of the institutional research committee and with the 1964 Helsinki declaration and its later amendments or comparable ethical standards.

All methods were carried out in accordance with relevant guidelines and regulations.

\section{References}

1. Bray F, Ferlay J, Soerjomataram I, et al. (2018) Global cancer statistics 2018: GLOBOCAN estimates of incidence and mortality worldwide for 36 cancers in 185 countries. CA Cancer J Clin 68(6):394424

2. Lafaro KJ, Demirjian AN, Pawlik TM (2015) Epidemiology of Hepatocellular Carcinoma. Surg Oncol Clin N Am 24:1-17

3. Gheorghe G, Stoian AP, Gaman M-A, et al. (2019) The Benefits and Risks of Antioxidant Treatment in Liver Diseases. Revista de Chimie 70:651-655.

4. Murakami T, Ishimaru $\mathrm{H}$, Sakamoto I, et al. (2007) Percutaneous radiofrequency ablation and transcatheter arterial chemoembolization for hypervascular hepatocellular carcinoma: rate and risk factors for local recurrence. Cardiovasc Intervent Radiol 30(4):696-704.

5. Bruix J, Castells A, Bosch J, et al. (1996) Surgical resection of hepatocellular carcinoma in cirrhotic patients: prognostic value of preoperative portal pressure. Gastroenterology 111(4):1018-1022. 
6. Marrero JA, Kulik LM, Sirlin CB, et al. (2018) Diagnosis, Staging, and Management of Hepatocellular Carcinoma: 2018 Practice Guidance by the American Association for the Study of Liver Diseases. Hepatology 68(2):723-750

7. Chernyak V, Fowler KJ, Kamaya A, et al. (2018) Liver Imaging Reporting and Data System (LI-RADS) Version 2018: Imaging of Hepatocellular Carcinoma in At-Risk Patients. Radiology 289(3):816-830

8. Ryder SD (2003) Guidelines for the diagnosis and treatment of hepatocellular carcinoma (HCC) in adults. Gut 52 Suppl 3:iii $1-8$.

9. Hirooka M, Hiraoka A, Ochi H, et al. (2018) Transcatheter Arterial Chemoembolization With or Without Radiofrequency Ablation: Outcomes in Patients With Barcelona Clinic Liver Cancer Stage B Hepatocellular Carcinoma. AJR Am J Roentgenol 210(4):891-898.

10. Kim W, Cho SK, Shin SW, et al. (2019) Combination therapy of transarterial chemoembolization (TACE) and radiofrequency ablation (RFA) for small hepatocellular carcinoma: comparison with TACE or RFA monotherapy. Abdominal Radiology 44(6):2283-2292.

11. Ren Y, Cao Y, Ma H, et al. (2019) Improved clinical outcome using transarterial chemoembolization combined with radiofrequency ablation for patients in Barcelona clinic liver cancer stage A or B hepatocellular carcinoma regardless of tumor size: results of a single-center retrospective case control study. BMC Cancer 19(1):983.

12. Llovet JM, Bru C, Bruix J (1999) Prognosis of hepatocellular carcinoma: the BCLC staging classification. Semin Liver Dis 19(3):329-338.

13. Yamakado K, Hirota S (2015) Sub-classification of intermediate-stage (Barcelona Clinic Liver Cancer stage-B) hepatocellular carcinomas. World J Gastroenterol 21(37):10604-10608.

14. Pawlik TM, Delman KA, Vauthey JN, et al. (2005) Tumor size predicts vascular invasion and histologic grade: Implications for selection of surgical treatment for hepatocellular carcinoma. Liver Transpl 11(9):1086-1092.

15. Hsieh CH, Wei CK, Yin WY, et al. (2015) Vascular invasion affects survival in early hepatocellular carcinoma. Mol Clin Oncol 3(1):252-256.

16. Vauthey JN, Lauwers GY, Esnaola NF, et al. (2002) Simplified staging for hepatocellular carcinoma. J Clin Oncol 20(6):1527-1536.

17. Zhang B, Moser MAJ, Zhang EM, et al. (2014) Study of the relationship between the target tissue necrosis volume and the target tissue size in liver tumors using two-compartment finite element RFA modelling. Int J Hyperthermia 30(8):593-602

18. Kim DJ, Clark PJ, Heimbach J, et al. (2014) Recurrence of hepatocellular carcinoma: importance of mRECIST response to chemoembolization and tumor size. Am J Transplant 14(6):1383-1390.

19. Nagao T, Inoue S, Goto $S$, et al. (1987) Hepatic resection for hepatocellular carcinoma. Clinical features and long-term prognosis. Ann Surg 205(1):33-40.

20. Khan KN, Yatsuhashi H, Yamasaki K, et al. (2000) Prospective analysis of risk factors for early intrahepatic recurrence of hepatocellular carcinoma following ethanol injection. J Hepatol 32(2):269278. 
21. Pote N, Cauchy F, Albuquerque M, et al. (2015) Performance of PIVKA-II for early hepatocellular carcinoma diagnosis and prediction of microvascular invasion. J Hepatol 62(4):848-854.

22. Nishie A, Yoshimitsu K, Okamoto D, et al. (2013) CT prediction of histological grade of hypervascular hepatocellular carcinoma: utility of the portal phase. Jpn J Radiol 31(2):89-98.

23. Okamoto D, Yoshimitsu K, Nishie A, et al. (2012) Enhancement pattern analysis of hypervascular hepatocellular carcinoma on dynamic MR imaging with histopathological correlation: validity of portal phase imaging for predicting tumor grade. Eur J Radiol 81(6):1116-1121.

24. Takenaka K, Adachi E, Nishizaki T, et al. (1994) Possible multicentric occurrence of hepatocellular carcinoma: a clinicopathological study. Hepatology 19(4):889-894.

25. Nakashima O, Kojiro M (2001) Recurrence of hepatocellular carcinoma: multicentric occurrence or intrahepatic metastasis? A viewpoint in terms of pathology. J Hepatobiliary Pancreat Surg 8(5):404409.

26. Johnson PJ, Berhane S, Kagebayashi C, et al. (2015) Assessment of liver function in patients with hepatocellular carcinoma: a new evidence-based approach-the ALBI grade. J Clin Oncol 33(6):550558.

27. Song MJ, Bae SH, Lee JS, et al. (2016) Combination transarterial chemoembolization and radiofrequency ablation therapy for early hepatocellular carcinoma. Korean J Intern Med 31(2):242252.

28. Sahara S, Kawai N, Tanaka T, et al. (2012) Prospective evaluation of transcatheter arterial chemoembolization (TACE) with multiple anti-cancer drugs (epirubicin, cisplatin, mitomycin c, 5fluorouracil) compared with TACE with epirubicin for treatment of hepatocellular carcinoma. Cardiovasc Intervent Radiol 35(6):1363-1371.

29. Hasegawa K, Kokudo N, Makuuchi M, et al. (2013) Comparison of resection and ablation for hepatocellular carcinoma: a cohort study based on a Japanese nationwide survey. J Hepatol 58(4):724-729.

30. Lewis S, Peti S, Hectors SJ, et al. (2019) Volumetric quantitative histogram analysis using diffusionweighted magnetic resonance imaging to differentiate $\mathrm{HCC}$ from other primary liver cancers. Abdom Radiol 44:912-922

\section{Figures}




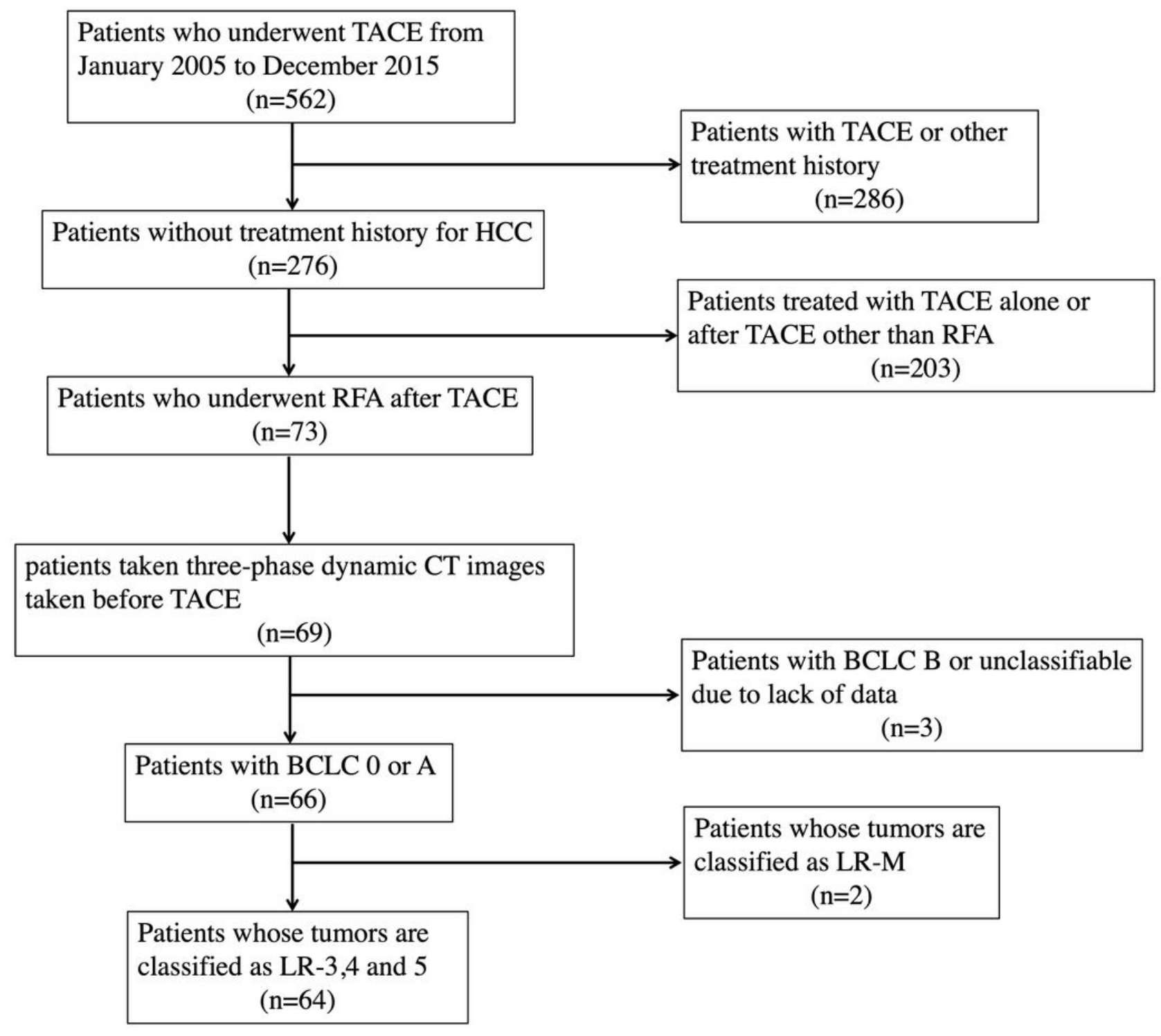

Figure 1

Survey population flow chart of patients with hepatocellular carcinoma (HCC). TACE: transcatheter arterial chemoembolization; RFA: radiofrequency ablation; CT: computed tomography; BCLC: BarcelonaClinic Liver Cancer Stage. 
a

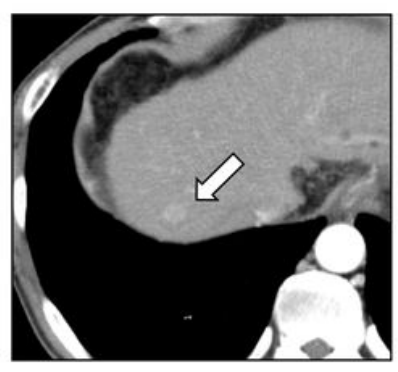

b

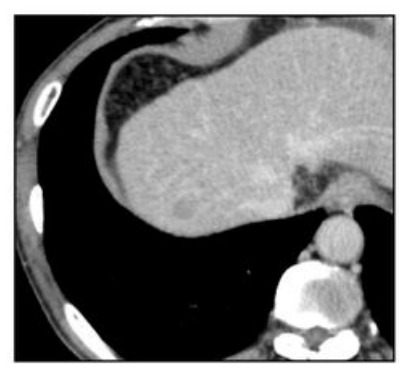

c

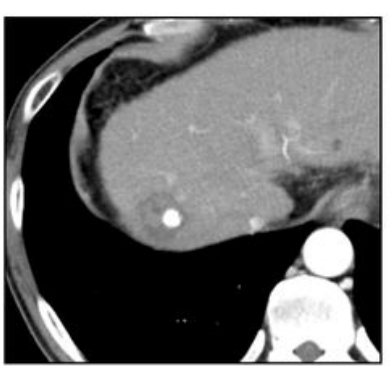

$\mathrm{d}$

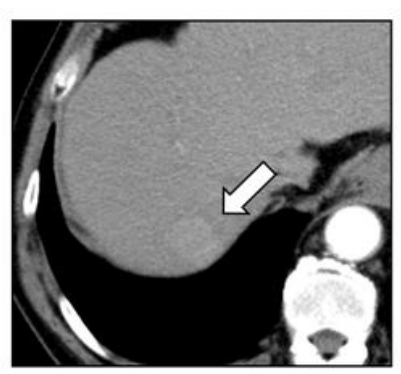

e

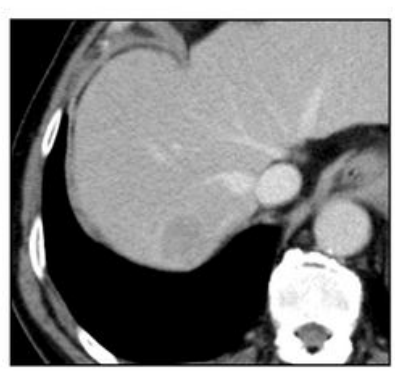

$\mathrm{f}$

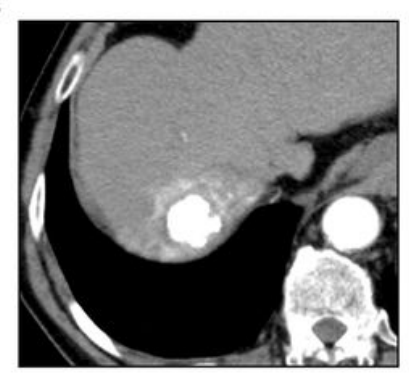

$\mathrm{g}$

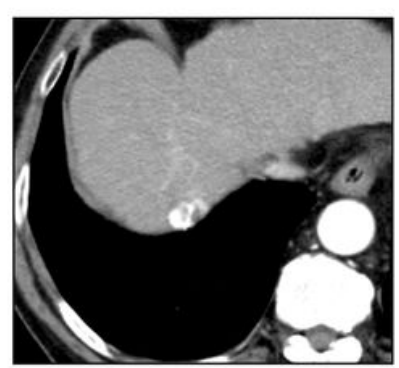

Figure 2

Clinical cases of HCC in patients with LR-4 and -5 .

a) LR-4 tumor in a 68-year-old man with HCC in the right lobe of the liver. Arterial phase of the contrastenhanced CT image before TACE. Arterial phase hyperenhancement measuring $9 \mathrm{~mm}$ was confirmed in liver dome.

b) Delayed phase of the contrast-enhanced CT image showed washout of the contrast.

c) Arterial phase of the contrast-enhanced CT 4 months after TACE-RFA showed good lipiodol accumulation surrounded by RFA-related hypoattenuation area. No viable lesions were seen. No recurrence was observed after 5 years.

d) LR-5 tumor in a 70-year-old man with HCC in the right lobe of the liver. Arterial phase of the contrastenhanced CT image before TACE. Arterial phase hyperenhancement measuring $23 \mathrm{~mm}$ was seen in the liver dome.

e) Delayed phase of the contrast-enhanced CT image before TACE showed washout of the lipiodol. 
f) Arterial phase of the contrast-enhanced CT image 3 days after TACE-RFA showed good lipiodol accumulation surrounded by RFA-related hypoattenuation area.

g) Arterial phase of the contrast-enhanced CT image taken 8 months after TACE-RFA. Arterial enhancement was confirmed adjacent to the treated site, indicating tumor recurrence.

a

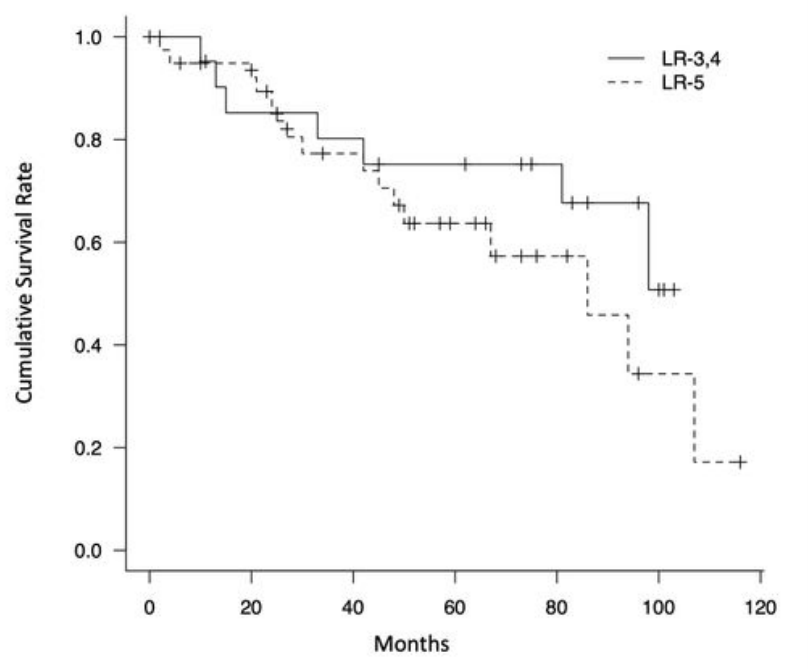

$\mathrm{b}$

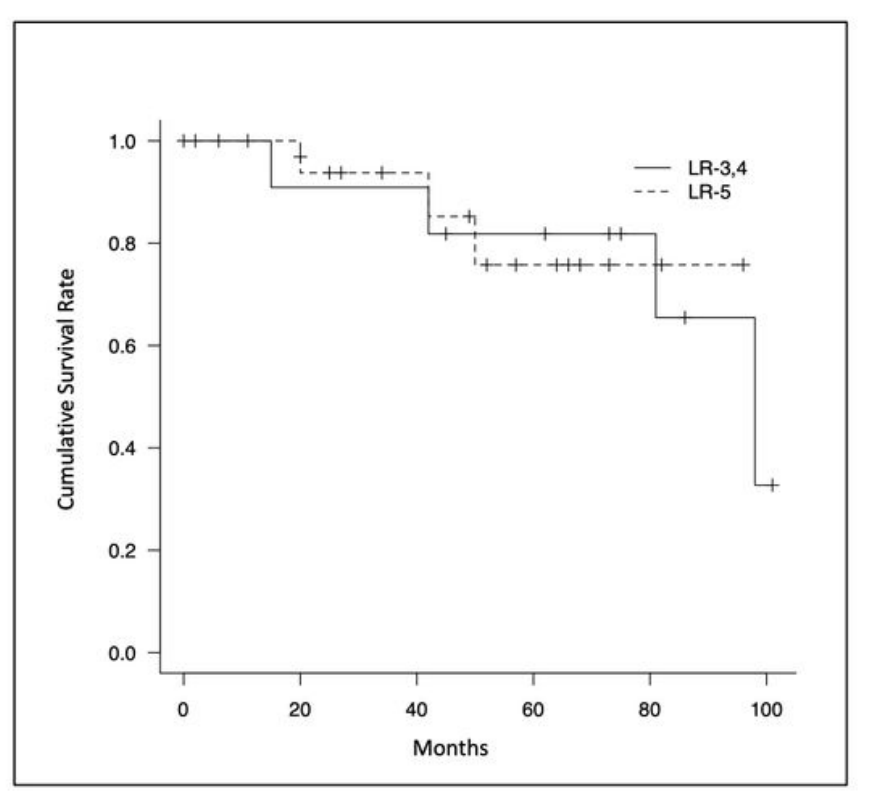

$\mathrm{c}$

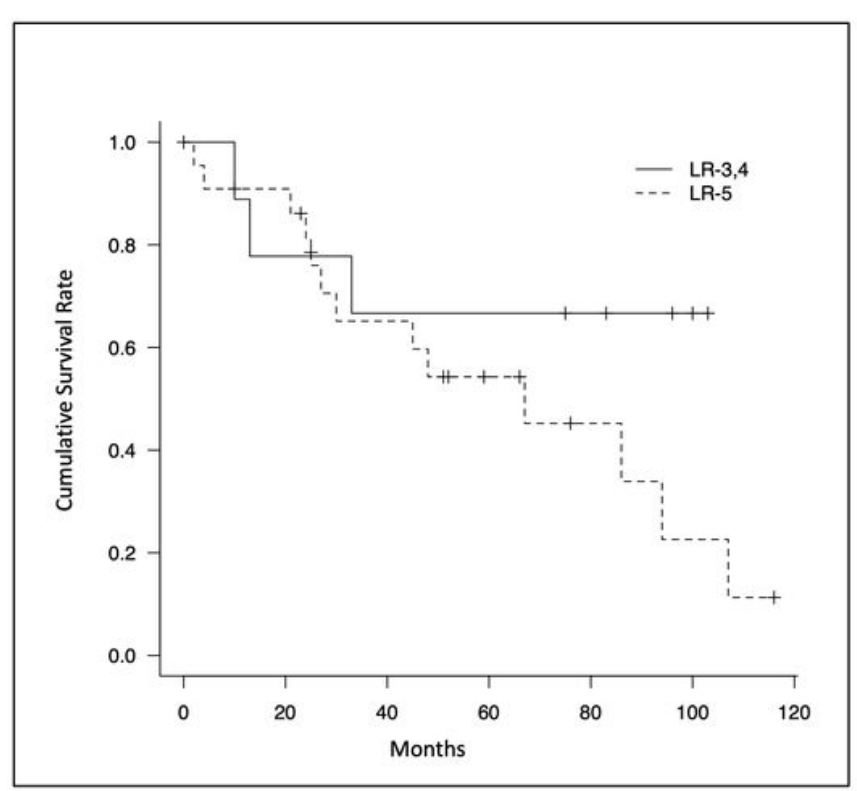

Figure 3

Kaplan-Meier curve comparing overall survival of LR-3 or -4 and LR-5. 
a) Comparison of LR-3 or -4 and LR-5 in all cases. There was no significant difference in this comparison $(p=0.278)$.

b) Comparison of LR-3 or -4 and LR- 5 in BCLC 0 . There was no significant difference in this comparison $(p=0.952)$.

c) Comparison of LR-3 or -4 and LR-5 in BCLC A. There was no significant difference in this comparison $(p=0.194)$.

a

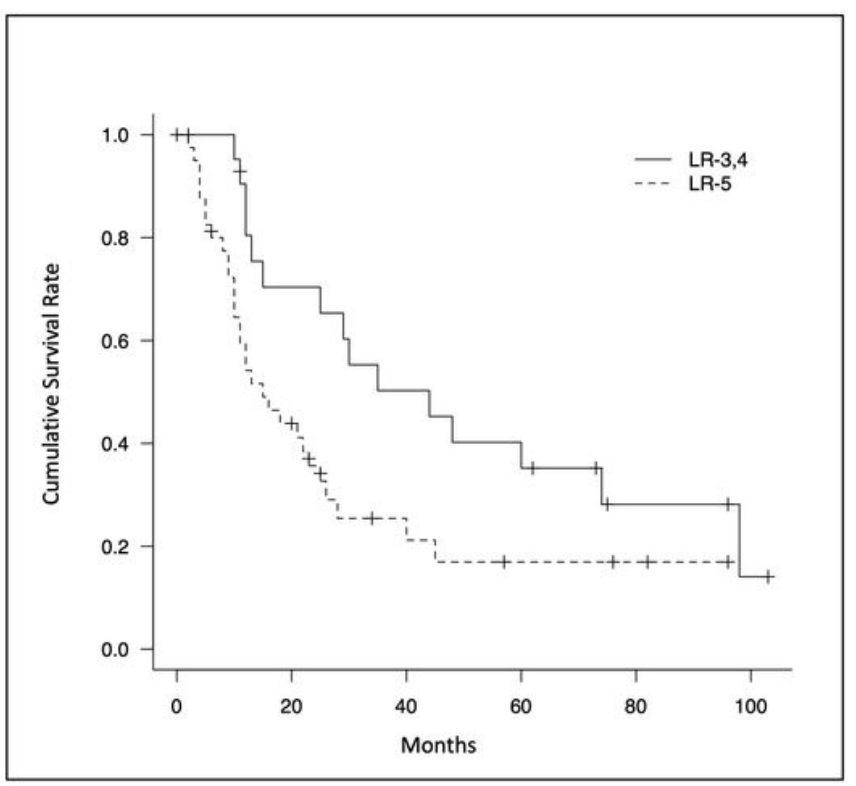

b

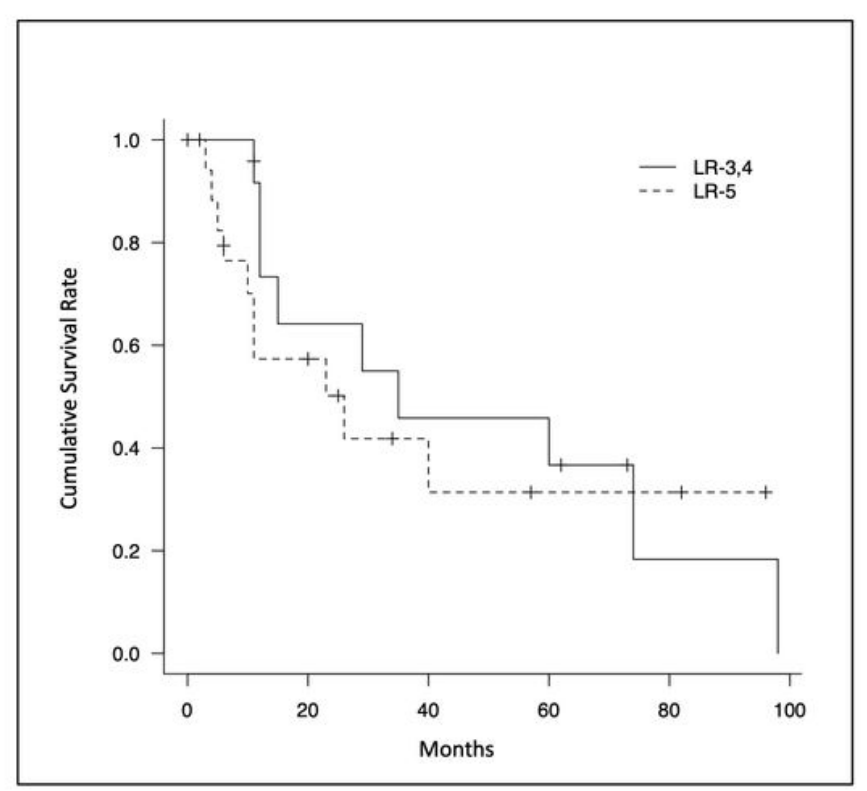

c

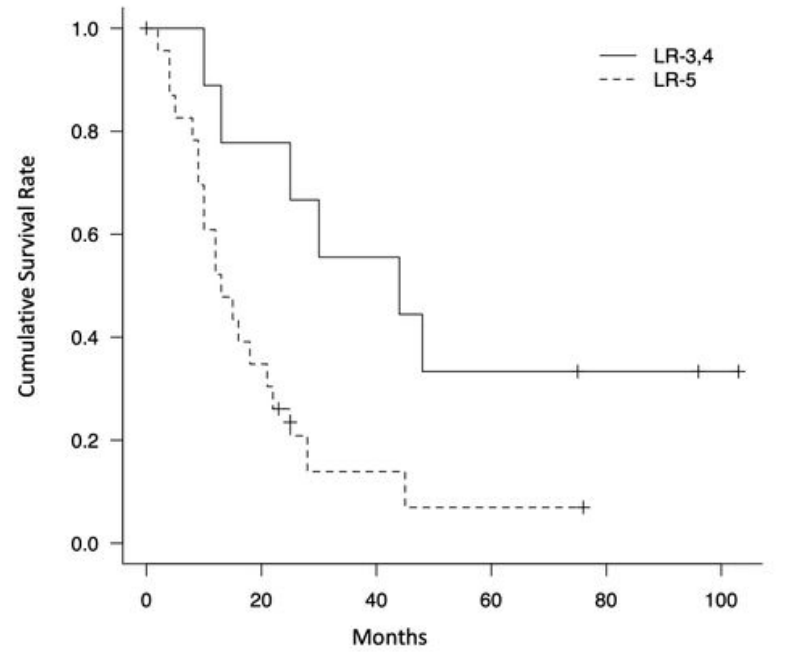




\section{Figure 4}

Kaplan-Meier curve comparing RFS of LR-3 or -4 and LR- 5 .

a) Comparison of LR-3 or -4 and LR-5 in all cases. There was a significant difference in this comparison $(p=0.030)$.

b) Comparison of LR-3 or -4 and LR-5 in BCLC 0 . There was no significant difference in this comparison $(p=0.533)$.

c) Comparison of LR-3 or -4 and LR-5 in BCLC A. There was a significant difference in this comparison ( $p$ $=0.016)$. 